\title{
Exploring functionality of the reverse $\beta$-oxidation pathway in Corynebacterium glutamicum for production of adipic acid
}

\author{
Jae Ho Shin ${ }^{1}$, Aaron John Christian Andersen ${ }^{2}$, Puck Achterberg ${ }^{1,3}$ and Lisbeth Olsson ${ }^{1 *}$ (i)
}

\begin{abstract}
Background: Adipic acid, a six-carbon platform chemical mainly used in nylon production, can be produced via reverse $\beta$-oxidation in microbial systems. The advantages posed by Corynebacterium glutamicum as a model cell factory for implementing the pathway include: (1) availability of genetic tools, (2) excretion of succinate and acetate when the TCA cycle becomes overflown, (3) initiation of biosynthesis with succinyl-CoA and acetyl-CoA, and (4) established succinic acid production. Here, we implemented the reverse $\beta$-oxidation pathway in C. glutamicum and assessed its functionality for adipic acid biosynthesis.

Results: To obtain a non-decarboxylative condensation product of acetyl-CoA and succinyl-CoA, and to subsequently remove CoA from the condensation product, we introduced heterologous 3-oxoadipyl-CoA thiolase and acyl-CoA thioesterase into C. glutamicum. No 3-oxoadipic acid could be detected in the cultivation broth, possibly due to its endogenous catabolism. To successfully biosynthesize and secrete 3-hydroxyadipic acid, 3-hydroxyadipylCoA dehydrogenase was introduced. Addition of 2,3-dehydroadipyl-CoA hydratase led to biosynthesis and excretion of trans-2-hexenedioic acid. Finally, trans-2-enoyl-CoA reductase was inserted to yield $37 \mathrm{\mu g} / \mathrm{L}$ of adipic acid.
\end{abstract}

Conclusions: In the present study, we engineered the reverse $\beta$-oxidation pathway in C. glutamicum and assessed its potential for producing adipic acid from glucose as starting material. The presence of adipic acid, albeit small amount, in the cultivation broth indicated that the synthetic genes were expressed and functional. Moreover, 2,3-dehydroadipyl-CoA hydratase and $\beta$-ketoadipyl-CoA thiolase were determined as potential target for further improvement of the pathway.

\section{Introduction}

The detrimental impact of petrochemical-based manufacturing on the environment has increased demand for biomass-derived chemicals, fuels, and consumer products [1, 2]. Biomass-based derived compounds now include fuels [3], monomers [4], polymers [5], and pharmaceuticals [2], with a few products already exerting a significant impact in the chemical industry [6, 7]. Global

*Correspondence: lisbeth.olsson@chalmers.se

${ }^{1}$ Department of Biology and Biological Engineering, Division of Industrial Biotechnology, Chalmers University of Technology, Gothenburg, Sweden

Full list of author information is available at the end of the article ongoing efforts are focused on expanding the portfolio, as well as increasing titers and yield.

Adipic acid, an aliphatic dicarboxylic acid, has gained wide attention from the metabolic engineering community as a six-carbon platform chemical for more than two decades [8]. Adipic acid is used as a building block for nylon-6,6, as well as other fibers, polyesters, and resins [9]. Its industrial production volume amounts to nearly 3 million US tons and is achieved via chemical oxidation of petroleum-derived KA oil (cyclohexanone and cyclohexanol) [10]. The petroleum-dependent nature of adipic acid production, has intensified the original author(s) and the source, provide a link to the Creative Commons licence, and indicate if changes were made. The images or other third party material in this article are included in the article's Creative Commons licence, unless indicated otherwise in a credit line to the material. If material is not included in the article's Creative Commons licence and your intended use is not permitted by statutory regulation or exceeds the permitted use, you will need to obtain permission directly from the copyright holder. To view a copy of this licence, visit http://creativecommons.org/licenses/by/4.0/. The Creative Commons Public Domain Dedication waiver (http://creativeco mmons.org/publicdomain/zero/1.0/) applies to the data made available in this article, unless otherwise stated in a credit line to the data. 
search for sustainable adipic acid precursors, derivatives, and analogues [11-13].

While bio-based production of adipic acid had been hampered by lack of efficient pathways, both synthetic [14-20] and naturally occurring pathways [21] have been characterized. Thermobifida fusca [21], as well as engineered Escherichia coli [14-20, 22], Saccharomyces cerevisiae [10], and Pseudomonas putida [23] can generate adipic acid from bio-based carbon sources, such as glucose or glycerol. As reviewed previously [9, 24-27], successful biosynthesis of adipic acid has often exploited the reverse $\beta$-oxidation pathway. This pathway is a synthetic extension of the tricarboxylic acid (TCA) cycle, has favorable thermodynamics $(-78.4 \mathrm{~kJ} / \mathrm{mol})$ [19], and leads to biosynthesis of coenzyme A (CoA)-activated intermediates via condensation of TCA metabolites, succinyl-CoA and acetyl-CoA. Whereas glycerol is an especially good carbon source for the production of adipic acid via reverse $\beta$-oxidation [15], the same has not been demonstrated for glucose. Reverse $\beta$-oxidation genes from combinatorial genetic sources have been applied in E. coli due to its relatively easy engineering [14, 16-19], whereas native adipic acid metabolic pathways have been identified in T. fusca [21]. Since the demonstration of adipic acid production from glucose in T. fusca, reverse $\beta$-oxidation genes from this bacterium have been applied in E. coli to attain high-titer adipic acid production from glycerol [15]. To further improve adipic acid titers from monosaccharides and ultimately lignocellulosic materials, new strategies are required.

Corynebacterium glutamicum is an established industrial workhorse for producing amino acids, such as L-glutamate [28] and L-lysine [29, 30]. New genetic engineering and bioinformatics tools have expanded the chemical product portfolio of C. glutamicum to include L-valine [31], pinene [32], diaminopentane [29], $\gamma$-aminobutyrate [33], 5-amiovaleric acid [34, 35], glutaric acid [36], alcohols [37], resveratrol [38], and muconic acid [39, 40]. Given that, unlike E. coli, C. glutamicum does not naturally consume glycerol [41], the majority of aforementioned studies on C. glutamicum have focused on simple sugars as carbon sources. Notably, bio-based production of 1,5-diaminopentane [42], 5-aminovaleric acid, and glutaric acid [43] has achieved higher titers in C. glutamicum than in E. coli [34-36, 44], likely due to higher metabolic flux toward amino acids in the former species [29]. Furthermore, engineered C. glutamicum can produce industrial titers of succinic acid (up to $146 \mathrm{~g} / \mathrm{L}$ ) [45], greatly surpassing other organisms [46]. Because the reverse $\beta$-oxidation pathway begins with TCA cycle intermediates and extensive amounts of succinic acid can be generated in C. glutamicum [47], introduction of adipic acid biosynthesis pathways in this organism has been proposed here in this study.

In the present study, we explored the generation of adipic acid using C. glutamicum as proof-of-concept cell factory. While adipic acid production from glucose has been attempted mostly in E. coli [14-20], development of other organisms may lead to potential improvements. To this end, we designed a synthetic pathway consisting of five heterologous genes (Fig. 1) and implemented reverse $\beta$-oxidation in $C$. glutamicum using glucose as carbon source. According to the designed scheme, E. coli 3-oxoadipyl-CoA thiolase (Paa); E.C. 2.3.1.174) $[14,16,19]$ catalyzes the condensation of acetyl-CoA and succinyl-CoA to form a six-carbon backbone. Next, the resulting 3-oxoadipyl-CoA is reduced by E. coli 3-hydroxyacyl-CoA dehydrogenase (PaaH; E.C. 1.1.1.157), and then dehydrated by $E$. coli 2,3-dehydroadipyl-CoA hydratase (PaaF; E.C. 4.2.1.17) [16, 19]. Subsequently, reduction by trans-2-enoyl-CoA reductase (Ter; E.C. 1.3.1.44) from Treponema denticola [48] is followed by release of CoA via thioesterase (TesB) from Acinetobacter baylyi [49] to finally yield adipic acid (Fig. 1).

\section{Results}

Assessment of non-decarboxylative Claisen condensation of acetyl-CoA and succinyl-CoA in C. glutamicum by overexpressing paaJ from $E$. coli

The design of synthetic pathway (Fig. 1) is motived by the similarities found in the bacterial phenylalanine catabolism pathways. Catabolism of phenylalanine in $E$. coli and styrene in Pseudomonas proceed via phenylacetate degradation [50-52]. In the last steps of phenylacetate catabolism, 2,3-dehydroadipyl-CoA is hydrated to 3-hydroxyadipyl-CoA, which is then oxidized to yield 3-oxoadipyl-CoA. Subsequently, succinyl-CoA and acetyl-CoA formed by thiolytic cleavage of 3-oxoadipylCoA are assimilated in the TCA cycle. Overexpression of $E$. coli paaF, paaH, and paaJ genes involved in these steps previously has demonstrated not only the reversibility of the individual enzymes, but also that of the pathway's direction $[16,19]$. Additionally, CoA-released byproducts of the abovementioned pathway, including 3-oxoadipate, 3-hydroxyadipate, and trans-2-hexenedioate, have been observed in the cultivation broth of $E$. coli overexpressing paaJ, paaH, and paaF [19]. While reverse $\beta$-oxidation may function through multiple cycles [53], PaaJ was shown to stop after the first cycle when overexpressed in E. coli, thus preventing the formation of $\mathrm{C} 8$ or C10 compounds [16]. Accordingly, here, E. coli PaaJ was chosen as the first reaction step (Fig. 1).

To assess whether non-decarboxylative Claisen condensation of succinyl-CoA and acetyl-CoA into 3-oxoadipyl-CoA occurred in C. glutamicum, the E. coli paaJ 


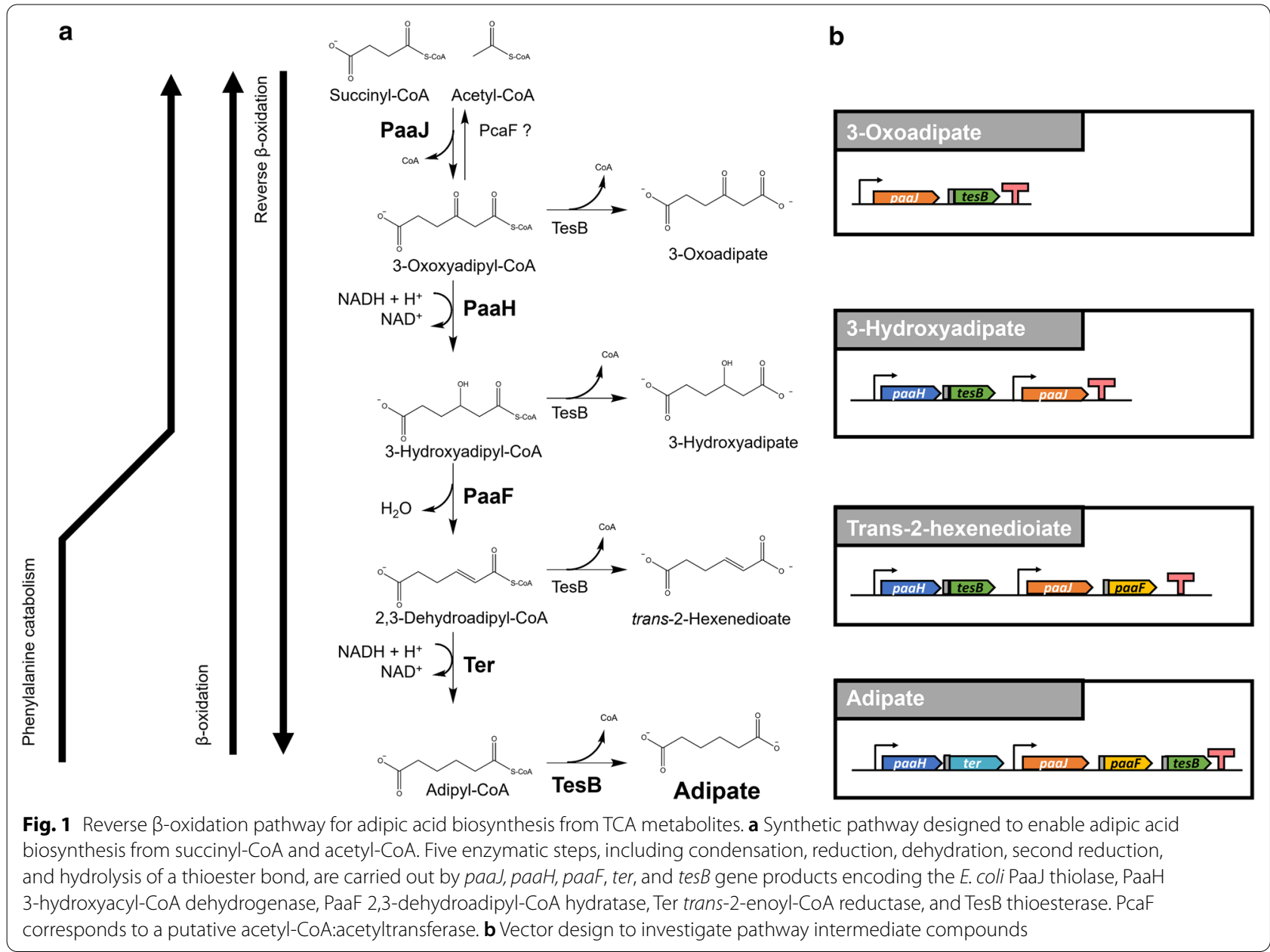

gene (KEGG ID: JW1392) was cloned into pZ8-Ptac (Fig. 1; Additional file 1: Table S1, Additional file 2: Table S2), an E. coli-C. glutamicum shuttle vector [54], and the CoA-released byproduct of 3-oxoadipyl-CoA was measured. TesB from $A$. baylyi is thought to have a broad substrate specificity and is known to release CoA from 3-oxoadipyl-CoA [19]. Similarly, E. coli thioesterase, which shares $43 \%$ identity with $A$. baylyi TesB, has been shown to release adipic acid from adipyl-CoA at its endogenous basal expression level [16]. Thus, tes $B$ from A. baylyi and paaJ from E. coli were organized in an operon behind the native tac promoter in the pZ8paaJ-tesB vector (Fig. 1; Additional file 1: Table S1). C. glutamicum was transformed with pZ8-paa)-tesB and its growth and capability to synthesize 3-oxoadipic acid by flask-batch cultivation were assessed (Fig. 2a). C. glutamicum harboring pZ8-paal-tesB reached $\mathrm{OD}_{600}$ of 26.4 after $72 \mathrm{~h}$. The metabolites excreted in the cultivation broth were derivatized with $O$-methylhydroxylamine (MeOX), and $N$-methyl- $N$-(trimethylsilyl)trifluoroacetamide (MSTFA), and analyzed by GC/MS. As 3-oxoadipic acid could not be detected (Additional file 3: Fig. S1), we speculated that it was metabolized and, instead, decided to measure the next byproduct of the pathway.

\section{Demonstration of 3-hydroxyadipate biosynthesis from engineered $C$. glutamicum}

To biosynthesize and detect 3-hydroxyadipate (Fig. 1), we cloned $p a a J$, tes $B$, and a codon-optimized version of E. coli paaH (KEGG ID: JW1390) in the pZ8-Ptac backbone. 3-hydroxyadipate has previously been detected in recombinant $E$. coli [19]. To ensure sufficient expression of paaJ, tesB, and paaH from a vector-based operon in C. glutamicum [35], the new pZ8-paaH-tesB-paaJ plasmid included an extra tac promoter [55]. The latter was added to the native tac promoter upstream of paaJ to ensure expression of paaJ and paaH. Owing to possible activity of endogenous $C$. glutamicum thioesterases, the tes $B$ gene accompanied by an additional ribosomebinding site (RBS) was placed downstream of $p a a H$. C. glutamicum transformed with pZ8-paaH-tesBpaaJ reached $\mathrm{OD}_{600}$ of 24.3 after $72 \mathrm{~h}$ of flask-batch 

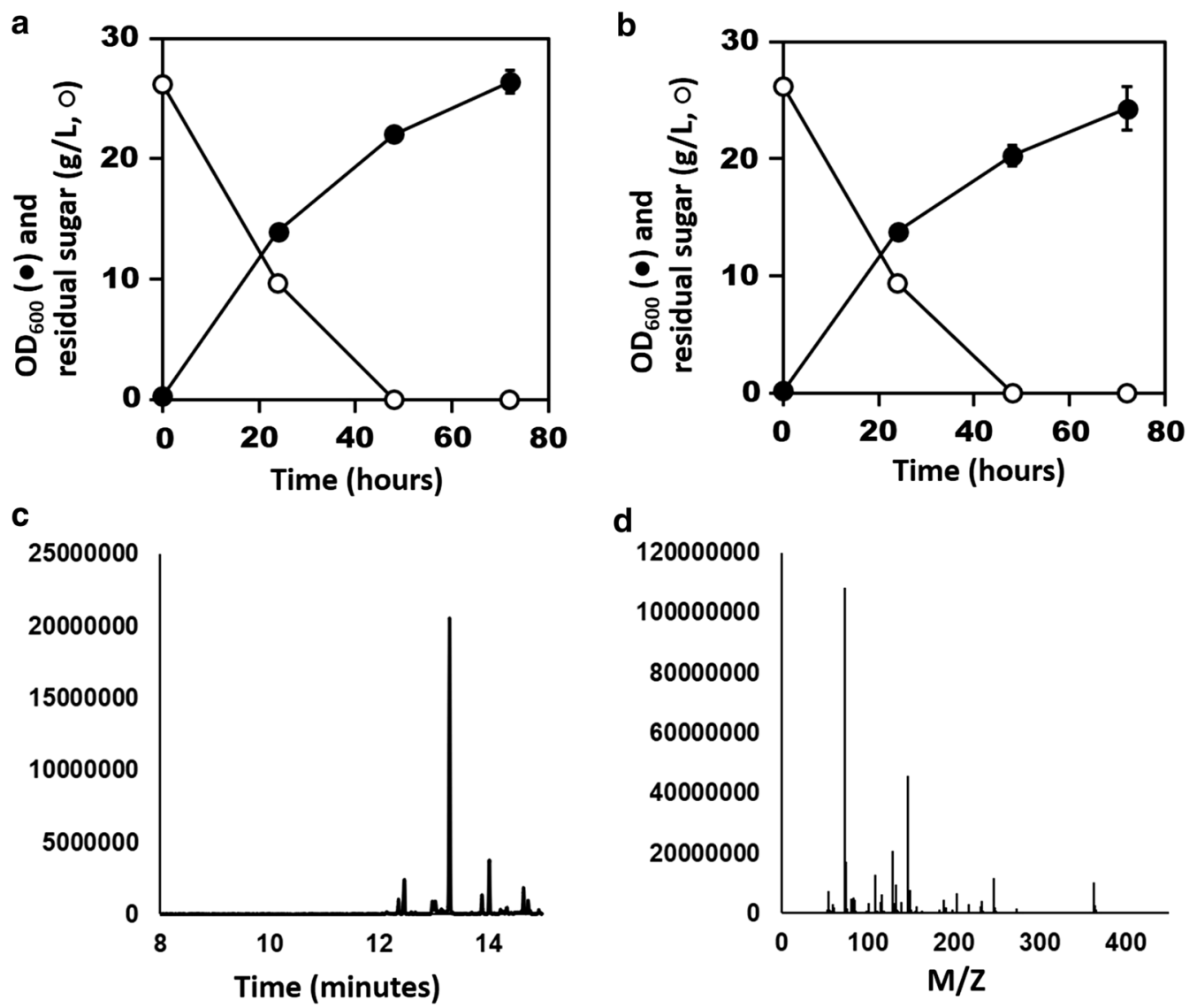

Fig. 2 Biosynthesis of adipic acid pathway intermediates from glucose. Shake-flask cultivation profile of C. glutamicum harboring (a) pZ8-paaJ-tesB and (b) pZ8-paaH-tesB-paaJ. OD 600 (filled circles), residual sugar (empty circles). N=3; error bars=standard deviation. $\mathbf{c}$ Representative GC/MS ion $(\mathrm{m} / \mathrm{z}=363)$ extracted chromatogram of MSTFA-derivatized cultivation broth from C. glutamicum transformed with pZ8-paaH-tesB-paaJ. The peak at retention time 13.3 min corresponds to MSTFA-derivatized 3-hydroxyadipic acid. (d) $\mathrm{m} / \mathrm{z}$ fragmentation pattern of the peak corresponding to MSTFA-derivatized 3-hydroxyadipic acid from (c)

cultivation (Fig. 2b). MSTFA derivatization of the cultivation broth followed by GC/MS revealed the presence of 3-hydroxyadipic acid among excreted metabolites (Fig. 2c, d). The retention time of trimethylsilyl-3-hydroxyadipic acid was 13.3 min under our experimental conditions. The $m / z$ pattern of the compound matched that of the MSTFA-reacted 3-hydroxyadipic acid standard (Additional file 4: Fig. S2). These findings demonstrated successful heterologous overexpression of paaJ, $p a a H$, and tesB, resulting in production and excretion of 3-hydroxyadipate from engineered C. glutamicum. Moreover, the presence of 3-hydroxyadipic acid in the cultivation broth indirectly confirmed the correct functioning of PaaJ and condensation of acetyl-CoA and succinyl-CoA in engineered C. glutamicum. Thus, overexpression of PaaJ in C. glutamicum allows manipulation of the thermodynamic equilibrium between acetyl-CoA, succinyl-CoA, and 3-oxoadipyl-CoA, in spite of endogenous $\beta$-ketoadipyl CoA thiolase (PcaF), which is thought to promote the catabolic direction of the pathway [56].

\section{Demonstration of trans-2-hexenedioic acid from engineered $C$. glutamicum}

For trans-2-hexenedioic acid biosynthesis in C. glutamicum, PaaF (KEGG ID: b1393) from E. coli [50] was added to pZ8-paaH-tesB-paaJ. The resulting vector was named pZ8-paaH-tesB-paajF and transformed into $C$. glutamicum. PaaF from E. coli catalyzes the dehydration of 3-hydroxyadipyl-CoA [16, 19], forming 2,3-dehydroadipyl-CoA. The CoA-released form of the latter compound, trans-2-hexenedioic acid, has been detected from an engineered E. coli strain [19].

The engineered C. glutamicum strain expressing the four genes reached $\mathrm{OD}_{600}$ of 24.8 after $72 \mathrm{~h}$ shake-flask cultivation (Fig. 3a). Concentrating the cultivation broth 5 -fold using solid-phase extraction followed by LC/MS revealed presence of trans-2-hexenedioic acid (Fig. 3b). 

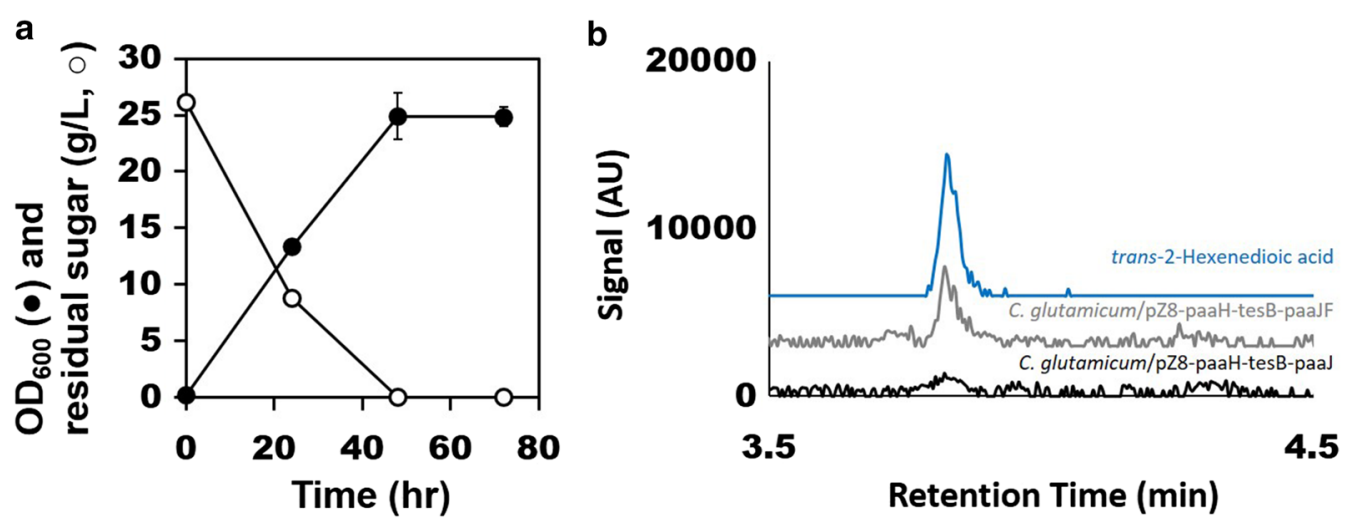

Fig. 3 Shake-flask cultivation profile of C. glutamicum harboring pZ8-paaH-tesB-paaJF. a Cultivation profile including $\mathrm{OD}_{600}$ (filled circle) and residual sugar (empty circle). $N=3$; error bars = standard deviation. $\mathbf{b}$ lon $(\mathrm{m} / \mathrm{z}=143.03498)$ extracted chromatogram obtained by $\mathrm{LC} / \mathrm{MS}$ of trans-2-hexenedioic acid (blue), cultivation broth of C. glutamicum harboring pZ8-paaH-tesB-paaJF (grey), and a control vector (black)

C. glutamicum strain harboring pZ8-paaH-tesB-paaJ did not produce detectable amount of trans-2-hexenedioic acid (Fig. 3b). The results indirectly demonstrated expression of paaJ, paaH, paaF, and tesB genes despite the operon organization with placing $p a a F$ and $t e s B$ at the downstream of each operon (Fig. 1). Downstream genes are known to be expressed weaker than the upstream genes in operon configurations [57]. However, the results also suggested that either expression of operons was not optimal for downstream genes or there was a bottleneck in the pathway.

\section{Implementation of full-length reverse $\beta$-oxidation in $C$. glutamicum}

Addition of Ter from T. denticola [48] completed the reverse $\beta$-oxidation pathway for adipic acid production in C. glutamicum (Fig. 1). Overexpression of ter from T. denticola (KEGG ID: TDE0597) was shown to reduce trans-2-enoyl-CoA in engineered $E$. coli [16]. To express codon-optimized paaJ, paaH, paaF, ter, and tesB on a single vector, the genes were reorganized in two separate operons, each driven by a tac promoter and constructed in such a way that paa $F$ was expressed after paaJ and ter after $p a a H$. Expression of tes $B$ was again given the lowest priority and was placed in the last position of the second operon, downstream of paaF. Translation of paaF, ter, and tes $B$ was enabled by each accompanying synthetic RBS. C. glutamicum transformed with the resulting pZ8paaH-ter-paaJ-paaF-tesB plasmid reached $\mathrm{OD}_{600}$ of 25.2 after $78 \mathrm{~h}$ of flask-batch cultivation (Fig. 4a). MSTFA derivatization of the cultivation broth followed by $\mathrm{GC} /$ MS revealed the presence of 3-hydroxyadipate (Fig. 4b, c) but not trans-2-hexenedioic acid or adipic acid. However, concentrating cultivation broth 5 -fold followed by LC-MS revealed presence of $37.3 \mu \mathrm{g} / \mathrm{L}$ of adipic acid
(Fig. 4d). Analysis of LC-MS/MS further confirmed the identity of adipic acid from the cultivation broth (Additional file 5: Fig. S3). The MS/MS spectra obtained was also in agreement with publicly available database entry (HMDB0000448) [58]. C. glutamicum harboring an empty vector also reached $\mathrm{OD}_{600}$ of 25.2 (Fig. 4c), and no intermediate compounds were detected in the control strain (Fig. 4b-d). The presence of an intermediate and adipic acid confirmed that even when tesB expression was given the least priority, release of CoA was not hindered by the pZ8-paaH-ter-paaJ-paaF-tesB construct. A possible reason for this is that native thioesterase (i.e., NCgl1600) activity was strong enough to release CoA from 3-hydroxyadipyl-CoA or that expression and functionality of tesB were achieved. However, these results also pointed to the need for a new strategy to assess and improve gene expression of the attempted synthetic pathway.

\section{Discussion}

In this study, we aimed to investigate the reverse $\beta$-oxidation pathway and its functionality in engineered C. glutamicum. In the final strain, detection of 3-hydroxyadipic acid as major byproduct, small amount of adipic acid, and concomitant absence of trans2-hexenedioic acid pointed to inefficient PaaF activity, although Ter functionality should be assessed separately as well (Figs. 3, 4). Such pattern indicated that the dehydration of 3-hydroxyadipyl-CoA was the bottleneck step. The paaF and ter genes were each placed downstream in the respective operons (Fig. 1), since placing the upstream pathway genes in the upstream of operon organization has proven advantageous [34, 35]. This also might have led to decreased expression of paaF. Hence, a possible strategy to overcome the expression challenge is 

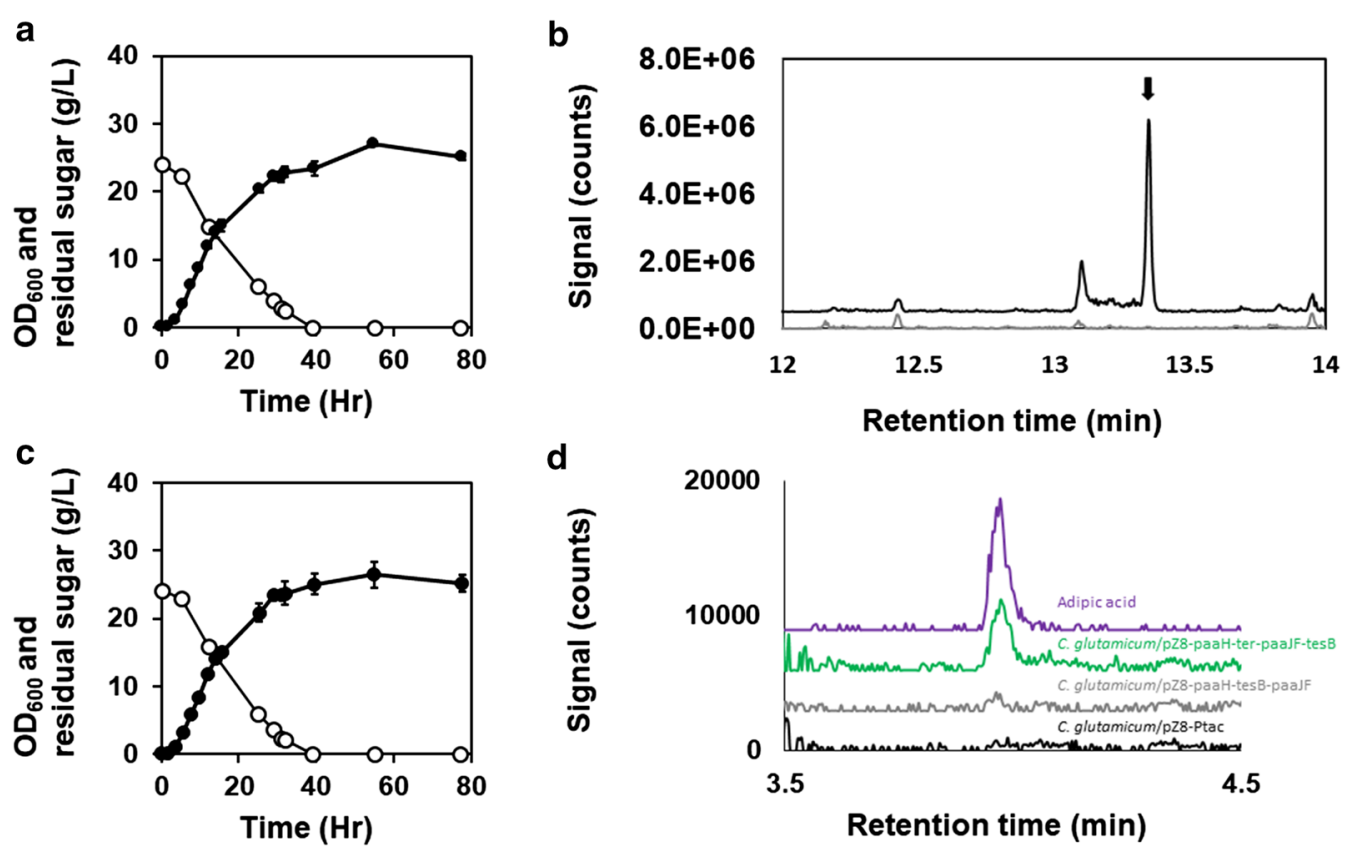

Fig. 4 Shake-flask cultivation profile of C. glutamicum harboring pZ8-paaH-ter-paaJ-paaF-tesB. a Cultivation parameters include $\mathrm{OD}_{600}$ (filled circles) and residual sugar (empty circles). $\mathrm{N}=3$; error bars = standard deviation. b lon $(\mathrm{m} / \mathrm{z}=363)$ extracted chromatogram obtained by GC/MS of the cultivation broth ( $t=55 \mathrm{~h}$ ) of C. glutamicum harboring pZ8-paaH-ter-paa--paaF-tesB (black line) and an empty vector (grey line). The peak corresponding to 3-hydroxyadipate is indicated with a black arrow. c Cultivation profile of C. glutamicum harboring pZ8-Ptac showing OD ${ }_{600}$ (filled circles) and residual sugar (empty circles). $N=3$; error bars = standard deviation. (d) lon $(m / z=145.0506)$ extracted chromatogram obtained by LC/ MS of adipic acid standard (purple), cultivation broth of C. glutamicum harboring pZ8-paaH-ter-paaJ-paaF-tesB (green), pZ8-paaH-tesB-paaJF (grey), and an empty vector (black)

to switch the operon organization or to place additional promoters for improved paaF and ter expression [57]. A potential strategy to overcome dehydration step is to use another enzyme, such as Ech (enoyl-CoA hydratase) from Ralstonia eutropha, Crt (crotonase) from Clostridium acetobutylicum [14], or Tfu_0067 from T. fusca [15], as it might be that $E$. coli PaaF is simply less compatible with C. glutamicum for some unknown reason.

Lack of 3-oxoadipic acid in the cultivation broth can be potentially explained by the presence of PcaF, which is encoded by cg2625 (EC:2.3.1.9) and involved in degradation of 3-oxoadipic acid [59]. Although paaJ overexpression might have allowed an equilibrium between 3-oxoadipyl-CoA, acetyl-CoA, and succinyl-CoA, PcaF could have represented a potential source of disturbance by reversing the direction of the chemical reaction. We speculate that PcaF acts against PaaJ to actively degrade 3-oxoadipyl-CoA. Furthermore, because 3-oxoadipic acid synthesized in the presence of PaaJ and TesB can be reverted to 3-oxoadipyl-CoA by PcaIJ [59], chromosomal deletion of pcaIJ and pcaF appears necessary to prevent degradation of 3-oxoadipyl-CoA and 3-oxoadipic acid.

Once bottleneck step of the dehydration of 3-hydroxyadipyl-CoA is overcome, further strategies to improve adipic acid production might include expression optimization by engineering the reductive branch of TCA cycle [60], RBS switching [61], alternating reducing equivalents between $\mathrm{NADH}^{+}$and NADPH [62], media engineering [63], eliminating competing pathways [64], using a different thioesterase for CoA removal [24], and removing any pathways leading to the formation of byproducts [65]. A switch in reducing equivalent can be achieved using trans-2-enoylCoA reductase (E.C. 1.3.1.38), which accepts NADPH, an abundant cofactor in C. glutamicum. Competing reaction can be eliminated by deleting $p c a F$, because the reverse degradation flux must be engineered to overcome the catabolic step [66]. Possible strain and pathway engineering strategies to improve titer and productivity have been discussed previously [67-69].

C. glutamicum offers various benefits, such as elevated tolerance to acids, strong TCA flux, and natural excretion of acetic and succinic acid upon metabolic stress. Its use of acetyl-CoA and succinyl-CoA in the initial steps was hypothesized to favor adipic acid production. However, while condensation of acetyl-CoA and succinyl-CoA was indeed successful, directing the flux toward adipic acid proved either impossible or insufficient. Hence, several 
challenges need to be overcome when engineering C. glutamicum for adipic acid production.

\section{Conclusions}

The present study demonstrated the feasibility of the synthetic reverse $\beta$-oxidation pathway by showcasing adipic acid biosynthesis in C. glutamicum. This work laid the groundwork for future development of C. glutamicum as a microbial factory for adipic acid production. For the subsequent studies, metabolic flux should be engineered to overcome the present bottleneck represented by dehydration of 3-hydroxyadipyl-CoA, while chromosomal deletion of catabolic genes will bring further advantages.

\section{Methods}

\section{Strains and plasmids}

Primers (Additional file 6: Table S3) used in this study were synthesized at Eurofins Genomics (Ebersberg, Germany), Thermo Fisher (Waltham, MA, USA) or SigmaAldrich (St. Louis, MO, USA). Codon-optimization for $C$. glutamicum (Additional file 2: Table S2) and subsequent gene synthesis were carried out at GenScript (Piscataway, NJ, USA). All constructed vectors were sequence-verified at Eurofins Genomics or Macrogen Europe (Amsterdam, the Netherlands). Plasmid pZ8-Ptac (Addgene plasmid number 74,064) was a gift from Timothy Lu (MIT, Cambridge, MA, USA) [54]. General PCR conditions were as follows: $95{ }^{\circ} \mathrm{C}$ for $5 \mathrm{~min}$; 28 cycles at $95{ }^{\circ} \mathrm{C}$ for $30 \mathrm{~s}$, $55{ }^{\circ} \mathrm{C}$ for $30 \mathrm{~s}, 72{ }^{\circ} \mathrm{C}$ for $1 \mathrm{~min}$; and a final extension at $72{ }^{\circ} \mathrm{C}$ for $7 \mathrm{~min}$. The PCR was carried out with Phusion High-Fidelity DNA Polymerase (Thermo Fisher) in a total reaction volume of $50 \mu \mathrm{L}$. All restriction enzymes were purchased from Thermo Fisher. E. coli NEB ${ }^{\circledR} 5$-alpha and NEB ${ }^{\circledR} 10$-beta from New England Biolabs (Ipswich, MA, USA) were used for general cloning purposes. The C. glutamicum ATCC 13,032 strain used in this study is a lab stock. Chemicals used in this study were purchased from Merck (Kenilworth, NJ, USA) or Thermo Fisher.

pZ8-paaI-tesB was constructed by cloning codon-optimized paaJ and tesB into the pZ8-Ptac vector. Primers 134_F_EcoRI and 133_i3R_KpnI v2 were used to amplify paaJ and the product was digested with EcoRI/KpnI. Primers tesB_F_KpnI and tesB_R_BamHI were used to amplify tes $B$ and the product was digested with KpnI/BamHI. pZ8-Ptac was digested with EcoRI/BamHI and subsequently three-way ligated with the two PCR products. A 16-bp RBS (tttcacacaggaaaca) from the synthetic lacUV5 promoter was placed between paaJ and tes $B$ for tes $B$ expression during this step.

Construction of pZ8-HB was carried out by replacing ter from pZ8-Hr with tes $B$. Codon-optimized tes $B$ was PCR-amplified with primers tesB_F_KpnI and tesB_R_BamHI, and then digested with $K p n \mathrm{I} /$ BamHI.
This fragment was ligated into pZ8-Hr previously digested with KpnI/BamHI to replace ter. An extra promoter and paaJ were amplified by primers PA146F_Gib and PA146R_Gib using pZ8-paaJ as template, and then inserted into pZ8-HB by Gibson assembly to yield pZ8-paaH-tesB-paaJ.

Trans-2-Hexenedioic acid producing vector, pZ8paaH-tesB-paaJF, was created by adding an extra promoter, paaJ, and paaF fragments to already prepared pZ8-HB. The promoter, paaJ, and paaF was PCR amplified using the primers, 143_F_Gibson and 143_R_Gib, and introduced into BamHI digested pZ8-HB.

Vector pZ8-paaH-ter-paaJ-paaF-tesB was constructed in a stepwise manner. A codon-optimized version of E. coli paaH was PCR-amplified using primers 133_i1F_EcoRI and 133_i1R_KpnI (Additional file 6: Table S3), and then digested with EcoRI and KpnI. A codon-optimized version of $T$. denticola ter was PCR-amplified using primers 133_i2F_KpnI and 133 i2R_BamHI and digested with KpnI and BamHI. The digested PCR products were three-way ligated with EcoRI/BamHI-digested pZ8-Ptac to yield pZ8_Hr. A 16-bp untranslated region (UTR) sequence (tttcacacaggaaaca) [55] including an RBS was introduced between the two genes for codon-optimized ter expression. To construct pZ8_HrJF, an extra tac promoter, codon-optimized paaJ, and paaF were introduced into pZ8_Hr. The codon-optimized version of paaJ was PCR-amplified using primers pre132_i3F_BamHI and 133_i3R_KpnI v2, and subsequently digested with $B a m \mathrm{HI} / K p n \mathrm{I}$. The codon-optimized paaF fragment (PCR-amplified with primers 133_i4F_KpnI and 133 i4R_PstI v2) was digested with KpnI/PstI. Digested paaJ and paaF fragments were subsequently threeway ligated into pZ8_Hr previously digested with BamHI/PstI. As with pZ8_Hr, an additional 16-bp UTR (tttcacacaggaaaca) with RBS was introduced at this point to allow for paaF expression. Thus, an extra promoter was PCR-amplified with primers tac_BamHI_F and tac_BamHI_R, digested with BamHI, and then ligated upstream of paaJ at the BamHI site. The resulting plasmid was named pZ8_HrJF. For pZ8-paaH-terpaaJ-paaF-tesB construction, the tes $B$ gene was added to pZ8_HrJF. Codon-optimized tesB was PCR-amplified using primers 133_i5F_PstI and 133_i5R_PstI, and then digested with Pst. The resulting fragment was ligated into pZ8_HrJF at the PstI site downstream of paaF.

pZ8-paal was constructed by removing tes $B$ from pZ8paaJ-tesB. This was achieved by digesting pZ8-paaJ-tesB with $K p n I$ and $B a m H I$, followed by ligation, whereby a short linker was placed at the $3^{\prime}$ end of paaJ as KpnI and BamHI were not compatible. The linker consisted of oligos linker_sense and linker_antisense (Additional file 6: 
Table S3). This step introduced also an XbaI site between $K p n I$ and BamHI for potential future usage.

\section{Pathway intermediate standards}

Standards of pure 3-oxoadipic acid (O856825) and 3-hydroxyadipic acid (H943100) were purchased from Toronto research chemicals (North York, ON, Canada). Trans-2-hexenedioic acid was synthesized by our group during a previous study [70].

\section{Media}

Pre-culture medium included (per liter): $40 \mathrm{~g}$ brain heart infusion, $10 \mathrm{~g}$ glucose, and $20 \mathrm{~g}$ D-sorbitol. Flask cultivation medium included (per liter): $25 \mathrm{~g}$ glucose, $2 \mathrm{~g}$ $\mathrm{MgSO}_{4} \cdot 7 \mathrm{H}_{2} \mathrm{O}, 1 \mathrm{~g} \mathrm{~K} \mathrm{HPO}_{4}, 1 \mathrm{~g} \mathrm{K \textrm {K } _ { 2 }} \mathrm{PO}_{4}, 1 \mathrm{~g}$ urea, $20 \mathrm{~g}$ $\left(\mathrm{NH}_{4}\right)_{2} \mathrm{SO}_{4}, 10 \mathrm{~g}$ yeast extract, $0.65 \mathrm{mg}\left(\mathrm{NH}_{4}\right)_{6} \mathrm{Mo}_{7} \mathrm{O}_{24}$, $7.726 \mathrm{mg} \mathrm{CaCl}{ }_{2} \cdot 2 \mathrm{H}_{2} \mathrm{O}, 11.35 \mu \mathrm{g}$ biotin, $4.57 \mathrm{mg}$ thiamin, $9 \mathrm{mg} \mathrm{FeSO} \cdot 7 \mathrm{H}_{2} \mathrm{O}, 7.88 \mathrm{mg} \mathrm{MnSO}{ }_{4} \cdot \mathrm{H}_{2} \mathrm{O}, 5.5 \mathrm{mg} \mathrm{CuSO}$. $5 \mathrm{H}_{2} \mathrm{O}, 10.06 \mathrm{mg} \mathrm{ZnSO} \cdot 7 \mathrm{H}_{2} \mathrm{O}$, and $1.88 \mathrm{mg} \mathrm{NiCl} \cdot 6 \mathrm{H}_{2} \mathrm{O}$. Additionally, $20 \mathrm{~g} / \mathrm{L}$ of $\mathrm{CaCO}_{3}$ was added to each flask as buffer and $\mathrm{CaCO}_{3}$ was removed by diluted $\mathrm{HCl}$ post-cultivation.

\section{Cultivation conditions}

Cultivation was carried out in 100-mL Erlenmeyer flasks at $30{ }^{\circ} \mathrm{C}$ and $200 \mathrm{rpm}$ in a shaking incubator (KS $4000 \mathrm{I}$ control; IKA-Werke GmbH, Staufen im Breisgau, Germany). Each strain was maintained in $60 \%$ glycerol solution and was inoculated in $5 \mathrm{~mL}$ pre-culture medium. After $17-19 \mathrm{~h}$, when $\mathrm{OD}_{600}$ reached 5.5-6.0, $1 \mathrm{~mL}$ of pre-culture was spun down at $3000 \mathrm{rcf}$ and the pellet was resuspended in $25 \mathrm{~mL}$ of cultivation medium. When applicable, isopropyl $\beta$-d-1-thiogalactopyranoside at a final concentration of $1 \mathrm{mM}$ was added at $\mathrm{OD}_{600} 0.5-0.8$. Neomycin at a final concentration of $50 \mu \mathrm{g} / \mathrm{L}$ was added as a selective marker during each cultivation.

\section{Cell growth and routine metabolite analysis}

Cell growth during cultivation was monitored by measuring $\mathrm{OD}_{600}$ on a Genesys 20 spectrophotometer (Thermo Fisher).

The concentration of glucose and organic acids in cultivation broth was analyzed by a Jasco (Tokyo, Japan) LC-4000 high-performance liquid chromatography system equipped with an autosampler (AS-4150), a pump (PU-4180), a column oven (CO-4061), a UV detector (RI-4030), and an RI detector (UV-4075). The mobile phase consisted of $5 \mathrm{mM} \mathrm{H}_{2} \mathrm{SO}_{4}$ and was pumped at constant flow rate of $0.8 \mathrm{~mL} / \mathrm{min}$. A Rezex ROA-organic acid $\mathrm{H}+$ column (Phenomenex, Torrance, CA, USA) maintained at $80{ }^{\circ} \mathrm{C}$ was used to separate the metabolites in the cultivation broth. The concentration of each compound was determined by Jasco ChomNAV software (version 2.03.03).

\section{Confirmation of 3-hydroxyadipic acid by GC/MS analysis}

3-hydroxyadipic acid was identified by GC/MS as reported previously with minor modifications [19, 71]. Briefly, $40 \mu \mathrm{L}$ of cell-free cultivation broth was lyophilized, resuspended in $50 \mu \mathrm{L}$ pyridine, and reacted with 50 $\mu \mathrm{L} \mathrm{MeOX}\left(20 \mathrm{~g} / \mathrm{L}\right.$ in pyridine) for $120 \mathrm{~min}$ at $30{ }^{\circ} \mathrm{C}$. The metabolites were then derivatized with $50 \mu \mathrm{L}$ MSTFA at $60{ }^{\circ} \mathrm{C}$ for $1 \mathrm{~h}$ prior to injection.

The GC/MS system was equipped with a Focus GC (Thermo Fisher), ISQ MS, and PAL COMBI-xt autosampler. A Zebron ZB-5MS column (Phenomenex) was used, with $1.1 \mathrm{~mL} / \mathrm{min}$ gas flow and helium as the carrier gas. The column temperature gradient was as follows: initial hold at $60{ }^{\circ} \mathrm{C}$ for $1 \mathrm{~min}$; ramp at $10^{\circ} \mathrm{C} / \mathrm{min}$ to $325^{\circ} \mathrm{C}$; and final hold at $325^{\circ} \mathrm{C}$ for $10 \mathrm{~min}$. Equilibration time was $0.5 \mathrm{~min}$ and preparatory run time out was $10 \mathrm{~min}$. The inlet temperature was $250{ }^{\circ} \mathrm{C}$. The electron multiplier was operated at $70 \mathrm{eV}$ with scanning acquisition mode. In total, $1 \mu \mathrm{L}$ of sample was injected for each analysis with split-less mode. The ion (MS) source was set at $250{ }^{\circ} \mathrm{C}$, mass filter (MS Quad) at $150{ }^{\circ} \mathrm{C}$, and MS transfer line at $290{ }^{\circ} \mathrm{C}$.

\section{Solid-phase extraction}

For sample clean up, $33 \mu \mathrm{m}$ polymeric reverse-phased solid-phase extraction (SPE) column $(200 \mathrm{mg} / 3 \mathrm{~mL}$; $8 \mathrm{~B}-\mathrm{S} 100-\mathrm{FBJ}$ ) was used with minor modification from manufacturer's protocols (Phenomenex). The precondition and equilibration steps were according to the manufacturer's protocols. The $5 \mathrm{~mL}$ of cell-free cultivation broth was pretreated with final $1 \%$ of formic acid prior to loading to the column. The column was washed with 1 $\mathrm{mL}$ water and eluted with $1 \mathrm{~mL}$ methanol. Methanol was removed by evaporation prior to next steps.

\section{Detection of trans-2-hexenedioic acid and adipic acid by LC-MS}

For detection of adipic acid and trans-2-hexenedioic acid, SPE treated samples were analyzed using LC-MS. A Dionex Ultimate 3000 RS UPLC with a diode array detector (DAD) was coupled to a Bruker Maxis QTOF MS with electrospray ionization (ESI) (Bruker Daltonics, Bremen, Germany). The system was equipped with a reversed phase column (Kinetex; $1.7 \mu \mathrm{m}, \mathrm{F} 5,100 \AA$, 150 $\mathrm{mm} \times 2.1 \mathrm{~mm}$; Phenomenex) and was maintained at $40{ }^{\circ} \mathrm{C}$. Gradient elution was utilized for separation and a constant flow rate of $400 \mu \mathrm{L} / \mathrm{min}$ was held throughout the run. Two eluents were used in the gradient elution: eluent A (H2O, $20 \mathrm{mM}$ formic acid) and eluent B (ACN, 20 $\mathrm{mM}$ formic acid). The gradient started with $10 \%$ eluent 
$B$ rising to $70 \%$ over $6.7 \mathrm{~min}$, followed by $70-100 \%$ eluent B over $1.3 \mathrm{~min}$, then held at $100 \%$ eluent B for $3 \mathrm{~min}$. Analysis was carried out in negative ESI. The scan range was $m / z 75-1250$ with 2 spectra per second. For the targeted fragmentation a collision energy of $15 \mathrm{eV}$ was used.

Prior to the analysis of samples, the QTOF-MS was calibrated using a sodium formate calibrant. In addition, all data files were recalibrated with an internal standard of sodium formate injected prior to initial sample elution for each sample.

\section{Supplementary Information}

The online version contains supplementary material available at https://doi.org/10. 1186/s12934-021-01647-7.

Additional file 1: Table S1. Strains and plasmids used in this study. Additional file 2: Table S2. Sequences of codon-optimized genes.

Additional file 3: Figure S1. GC/MS analysis of authentic 3-oxoadipic acid standard. (a) lon ( $m / z=318)$ extracted chromatogram and (b) $m / z$ fragmentation pattern for MeOX and MSTFA-derivatized 3-oxoadipic acid obtained by GC/MS. The inset in (b) corresponds to the GoLM metabolome database [72] entry (A166019) for the same compound. (c) Comparison of fragmentation pattern of 3-oxoadipic acid standard (upper) and database entry (lower).

Additional file 4: Figure S2. GC/MS analysis of authentic 3-hydroxyadipic acid standard. (a) lon-extracted $(m / z=363$ ) chromatogram of MSTFA-derivatized 3-hydroxyadipic acid standard. (b) $\mathrm{m} / \mathrm{z}$ fragmentation spectrum of 3-hydroxyadipate standard. (c) The NIST database entry (79677) for the same compound.

Additional file 5: Figure S3. LC-MS/MS analysis of adipic acid in the cultivation broth. (a) Extracted ion chromatogram ( $\mathrm{m} / \mathrm{z}$ 145.05) of cultivation broth of C. glutamicum harboring pZ8-paaH-ter-paaJ-paaF-tesB (green), pZ8- paaH-tesB-paaJF (grey), and an empty vector (black). Adipic acid standard is shown for comparison of retention time (purple). (b) MS/MS of $m / z 145.05$ of precursor ion $\left([\mathrm{M}-\mathrm{H}]^{-}\right)$from engineered $\mathrm{C}$. glutamicum (green) and adipic acid standard (purple).

Additional file 6: Table S3.Primers used in this study. Restriction enzyme sites are indicated in boldface. Extra RBS sequences for expression of the immediate downstream gene are underlined.

\section{Acknowledgements}

The authors would like to thank John Morrissey, Ph.D. (University College Cork), Andrey Grishin (University of Saskatchewan), and Robin Teufel Dr. rer. Nat. (University of Freiburg) for helpful discussions.

\section{Authors' contributions}

$\mathrm{LO}$ and JHS conceived the study and designed the experiments. JHS performed strain engineering, cultivation, and analytical experiments. PA assisted with molecular cloning and cultivation experiments. AJCA and JHS carried out LC-MS experiments and data analysis. LO and JHS analyzed the results and wrote the manuscript. All authors read and approved the final manuscript.

\section{Funding}

Open access funding provided by Chalmers University of Technology. LO and JHS acknowledge the support by the Swedish Research Council through Grant No. VR 2016-03344. PA is supported by an Erasmus scholarship.

\section{Availability of data and materials}

All data generated or analyzed during this study are included in this published article [and its supplementary information files].

\section{Declarations}

Ethics approval and consent to participate

Not applicable.

\section{Consent for publication}

Not applicable.

\section{Competing interests}

The authors declare that they have no competing interests.

\section{Author details}

${ }^{1}$ Department of Biology and Biological Engineering, Division of Industrial Biotechnology, Chalmers University of Technology, Gothenburg, Sweden. ${ }^{2}$ Department of Biotechnology and Biomedicine, Technical University of Denmark, Lyngby, Denmark. ${ }^{3}$ Present Address: Department of Biotechnology, Delft University of Technology, Delft, The Netherlands.

Received: 5 March 2021 Accepted: 29 July 2021

Published online: 04 August 2021

\section{References}

1. Straathof AJJ. Transformation of biomass into commodity chemicals using enzymes or cells. Chem Rev. 2014;114(3):1871-908.

2. Becker J, Wittmann C. Advanced biotechnology: metabolically engineered cells for the bio-based production of chemicals and fuels, materials, and health-care products. Angew Chemie - Int Ed. 2015;54(11):3328-50.

3. Rabinovitch-Deere CA, Oliver JWK, Rodriguez GM, Atsumi S. Synthetic biology and metabolic engineering approaches to produce biofuels. Chem Rev. 2013.

4. van Haveren J, Scott EL, Sanders J. Bulk chemicals from biomass. Biofuels Bioprod Biorefining. 2008;2(1):41-57.

5. Chen GQ, Patel MK. Plastics derived from biological sources: present and future: a technical and environmental review. Chem Rev. 2012.

6. Burgard A, Burk MJ, Osterhout R, Van Dien S, Yim H. Development of a commercial scale process for production of 1,4-butanediol from sugar. Curr Opin Biotechnol. 2016;42:118-25.

7. Barton NR, Burgard AP, Burk MJ, Crater JS, Osterhout RE, Pharkya P, et al. An integrated biotechnology platform for developing sustainable chemical processes. J Ind Microbiol Biotechnol. 2014;42(3):349-60.

8. Niu W, Draths KM, Frost JW. Benzene-free synthesis of adipic acid. Biotechnol Prog. 2002;18(2):201-11.

9. Polen T, Spelberg M, Bott M. Toward biotechnological production of adipic acid and precursors from biorenewables. J Biotechnol. 2013;167(2):75-84.

10. Raj K, Partow S, Correia K, Khusnutdinova AN, Yakunin AF, Mahadevan R. Biocatalytic production of adipic acid from glucose using engineered Saccharomyces cerevisiae. Metab Eng Commun. 2018;6:28-32.

11. Kruyer NS, Wauldron N, Bommarius AS, Peralta-Yahya P. Fully biological production of adipic acid analogs from branched catechols. Sci Rep. 2020;10(1)

12. Shi H, Zhang L, Wu Y, Yu R, Peng Y, Wang Y, et al. Production of adipic acid derivatives from d-glucaric acid by hydrodeoxygenation mediated with hydroiodic acid. Catal Letters. 2021;151(2):338-43.

13. Moon TS, Yoon SH, Lanza AM, Roy-Mayhew JD, Jones Prather KL. Production of glucaric acid from a synthetic pathway in recombinant Escherichia coli. Appl Environ Microbiol. 2009.

14. Yu J, Le, Xia XX, Zhong JJ, Qian ZG. Direct biosynthesis of adipic acid from a synthetic pathway in recombinant Escherichia coli. Biotechnol Bioeng. 2014;111(12):2580-6.

15. Zhao M, Huang D, Zhang X, Koffas MAG, Zhou J, Deng Y. Metabolic engineering of Escherichia coli for producing adipic acid through the reverse adipate-degradation pathway. Metab Eng. 2018;47:254-62.

16. Cheong S, Clomburg JM, Gonzalez R. Energy-and carbon-efficient synthesis of functionalized small molecules in bacteria using nondecarboxylative Claisen condensation reactions. Nat Biotechnol. 2016;34(5):556-61. 
17. Clomburg JM, Blankschien MD, Vick JE, Chou A, Kim S, Gonzalez R. Integrated engineering of $\beta$-oxidation reversal and $\omega$-oxidation pathways for the synthesis of medium chain $\omega$-functionalized carboxylic acids. Metab Eng. 2015;28:202-12.

18. Babu T, Yun EJ, Kim S, Kim DH, Liu KH, Kim SR, et al. Engineering Escherichia coli for the production of adipic acid through the reversed ß-oxidation pathway. Process Biochem. 2015;50(12):2066-71.

19. Kallscheuer N, Gätgens J, Lübcke M, Pietruszka J, Bott M, Polen T. Improved production of adipate with emopen Escherichia coli emclose by reversal of $\beta$-oxidation. Appl Microbiol Biotechnol. 2017:101:2371-82.

20. Sun J, Raza M, Sun X, Yuan Q. Biosynthesis of adipic acid via microaerobic hydrogenation of cis,cis-muconic acid by oxygen-sensitive enoate reductase. J Biotechnol. 2018;280:49-54.

21. Deng $Y$, Mao Y. Production of adipic acid by the native-occurring pathway in Thermobifida fusca B6. J Appl Microbiol. 2015;119(4):1057-63.

22. Zhou Y, Zhao M, Zhou S, Zhao Y, Li G, Deng Y. Biosynthesis of adipic acid by a highly efficient induction-free system in Escherichia coli. J Biotechnol. 2020.

23. Niu W, Willett H, Mueller J, He X, Kramer L, Ma B, et al. Direct biosynthesis of adipic acid from lignin-derived aromatics using engineered Pseudomonas putida KT2440. Metab Eng. 2020;59:151-61.

24. Skoog E, Shin JH, Saez-Jimenez V, Mapelli V, Olsson L. Biobased adipic acid - the challenge of developing the production host. Biotechnol Adv. 2018;36(8):2248-63.

25. Kruyer NS, Peralta-Yahya P. Metabolic engineering strategies to bio-adipic acid production. Curr Opin Biotechnol. 2017:45:136-43.

26. Bart JCJ, Cavallaro S. Transiting from adipic acid to bioadipic acid. Part II. Biosynthetic pathways. Ind Eng Chem Res. 2015;54(2):567-76.

27. Deng Y, Ma L, Mao Y. Biological production of adipic acid from renewable substrates: current and future methods. Biochem Eng J. 2016;105:16-26.

28. Momose H, Takagi T. Glutamic acid production in biotin-rich media by temperature-sensitive mutants of Brevibacterium lactofermentum, a novel fermentation process. Agric Biol Chem. 1978.

29. Becker J, Zelder O, Häfner S, Schröder H, Wittmann C. From zero to herodesign-based systems metabolic engineering of Corynebacterium glutamicum for I-lysine production. Metab Eng. 2011;13(2):159-68.

30. Becker J, Wittmann C. Bio-based production of chemicals, materials and fuelsCorynebacterium glutamicum as versatile cell factory. Curr Opin Biotechnol. 2012;23(4):631-640

31. Hasegawa S, Suda M, Uematsu K, Natsuma Y, Hiraga K, Jojima T, et al. Engineering of corynebacterium glutamicum for high-yield I-valine production under oxygen deprivation conditions. Appl Environ Microbiol. 2013;79(4):1250-7.

32. Kang MK, Eom JH, Kim Y, Um Y, Woo HM. Biosynthesis of pinene from glucose using metabolically-engineered Corynebacterium glutamicum. Biotechnol Lett. 2014.

33. Baritugo KA, Kim HT, David Y, Khang TU, Hyun SM, Kang KH, et al. Enhanced production of gamma-aminobutyrate (GABA) in recombinant Corynebacterium glutamicum strains from empty fruit bunch biosugar solution. Microb Cell Fact. 2018;17(1).

34. Rohles CM, Gießelmann G, Kohlstedt M, Wittmann C, Becker J. Systems metabolic engineering of Corynebacterium glutamicum for the production of the carbon-5 platform chemicals 5-aminovalerate and glutarate. Microb Cell Fact. 2016.

35. Shin JH, Park SH, Oh YH, Choi JW, Lee MH, Cho JS, et al. Metabolic engineering of Corynebacterium glutamicum for enhanced production of 5-aminovaleric acid. Microb Cell Fact. 2016;15(1).

36. Rohles CM, Gläser L, Kohlstedt M, Gießelmann G, Pearson S, Del Campo A, et al. A bio-based route to the carbon- 5 chemical glutaric acid and to bionylon-6,5 using metabolically engineered: corynebacterium glutamicum. Green Chem. 2018;20(20):4662-74.

37. Smith KM, Cho KM, Liao JC. Engineering Corynebacterium glutamicum for isobutanol production. Appl Microbiol Biotechnol. 2010;87(3):1045-55.

38. Kallscheuer N, Vogt M, Marienhagen J. A Novel synthetic pathway enables microbial production of polyphenols independent from the endogenous aromatic amino acid metabolism. ACS Synthetic Biol. 2017;6:410-5.

39. Becker J, Kuhl M, Kohlstedt M, Starck S, Wittmann C. Metabolic engineering of Corynebacterium glutamicum for the production of cis, cis-muconic acid from lignin. Microb Cell Fact. 2018;17(1).
40. Shin WS, Lee D, Lee SJ, Chun GT, Choi SS, Kim ES, et al. Characterization of a non-phosphotransferase system for cis,cis-muconic acid production in Corynebacterium glutamicum. Biochem Biophys Res Commun. 2018:499(2):279-84.

41. Rittmann D, Lindner SN, Wendisch VF. Engineering of a glycerol utilization pathway for amino acid production by Corynebacterium glutamicum. Appl Environ Microbiol. 2008.

42. Qian ZG, Xia XX, Lee SY. Metabolic engineering of Escherichia coli for the production of cadaverine: a five carbon diamine. Biotechnol Bioeng. 2011.

43. Park SJ, Kim EY, Noh W, Park HM, Oh YH, Lee SH, et al. Metabolic engineering of Escherichia coli for the production of 5-aminovalerate and glutarate as C5 platform chemicals. Metab Eng. 2013.

44. Kim HT, Baritugo KA, Oh YH, Hyun SM, Khang TU, Kang KH, et al. Metabolic engineering of Corynebacterium glutamicum for the high-level production of cadaverine that can be used for the synthesis of biopolyamide 510. ACS Sustain Chem Eng. 2018;6(4):5296-305.

45. Okino S, Noburyu R, Suda M, Jojima T, Inui M, Yukawa H. An efficient succinic acid production process in a metabolically engineered Corynebacterium glutamicum strain. Appl Microbiol Biotechnol. 2008;81(3):459-64.

46. Mazière $A$, Prinsen $P$, García $A$, Luque $R$, Len $C$. A review of progress in (bio) catalytic routes from/to renewable succinic acid. Bioproducts: Biofuels; 2017.

47. Briki A, Kaboré K, Olmos E, Bosselaar S, Blanchard F, Fick M, et al. Corynebacterium glutamicum, a natural overproducer of succinic acid? Eng Life Sci. 2020.

48. Tucci S, Martin W. A novel prokaryotic trans-2-enoyl-CoA reductase from the spirochete Treponema denticola. FEBS Lett. 2007;581 (8):1561-6.

49. Ukey R, Holmes WE, Bajpai R, Chistoserdov AY. Evaluation of thioesterases from Acinetobacter baylyi for production of free fatty acids. Can J Microbiol. 2017.

50. Teufel R, Mascaraque V, Ismail W, Voss M, Perera J, Eisenreich W, et al. Bacterial phenylalanine and phenylacetate catabolic pathway revealed. Proc Natl Acad Sci. 2010;107(32):14390-5.

51. Ismail W, El-Said Mohamed M, Wanner BL, Datsenko KA, Eisenreich W, Rohdich F, et al. Functional genomics by NMR spectroscopy: phenylacetate catabolism in Escherichia coli. Eur J Biochem. 2003:270(14):3047-54.

52. Nogales J, Macchi R, Franchi F, Barzaghi D, Fernández C, García JL, et al. Characterization of the last step of the aerobic phenylacetic acid degradation pathway. Microbiology. 2007;153(2):357-65.

53. Dellomonaco C, Clomburg JM, Miller EN, Gonzalez R. Engineered reversal of the $\beta$-oxidation cycle for the synthesis of fuels and chemicals. Nature. 2011;476(7360):355-9.

54. Cleto $\mathrm{S}$, Jensen JVK, Wendisch VF, LuTK. Corynebacterium glutamicum metabolic engineering with CRISPR interference (CRISPRi). ACS Synth Biol. 2016;5(5):375-85.

55. de Boer HA, Comstock $\sqcup$, Vasser M. The tac promoter: a functional hybrid derived from the trp and lac promoters. Proc Natl Acad Sci USA. 1983.

56. Shen XH, Zhou NY, Liu SJ. Degradation and assimilation of aromatic compounds by Corynebacterium glutamicum: another potential for applications for this bacterium? Appl Microbiol Biotechnol. 2012.

57. Lim HN, Lee Y, Hussein R. Fundamental relationship between operon organization and gene expression. Proc Natl Acad Sci USA. 2011;108(26):10626-31.

58. Wishart DS, Feunang YD, Marcu A, Guo AC, Liang K, Vázquez-Fresno R, et al. HMDB 4.0: the human metabolome database for 2018. Nucleic Acids Res. 2018.

59. Brinkrolf K, Brune I, Tauch A. Transcriptional regulation of catabolic pathways for aromatic compounds in Corynebacterium glutamicum. Genetics Mol Res GMR. 2006;5:773-89.

60. Hao T, Li G, Zhou S, Deng Y. Engineering the Reductive TCA Pathway to Dynamically Regulate the Biosynthesis of Adipic Acid in Escherichia coli. ACS Synth Biol. 2021.

61. Salis HM, Mirsky EA, Voigt CA. Automated design of synthetic ribosome binding sites to control protein expression. Nat Biotechnol. 2009.

62. Cahn JKB, Werlang CA, Baumschlager A, Brinkmann-Chen S, Mayo SL, Arnold FH. A General Tool for Engineering the NAD/NADP Cofactor Preference of Oxidoreductases. ACS Synth Biol. 2017.

63. Jeon JM, Thangamani R, Song E, Lee HW, Lee HW, Yang YH. Media optimization of Corynebacterium glutamicum for succinate production under oxygen-deprived condition. J Microbiol Biotechnol. 2013;23(2):211-7.

64. Kind S, Jeong WK, Schröder H, Zelder O, Wittmann C. Identification and elimination of the competing $\mathrm{N}$-acetyldiaminopentane pathway for 
improved production of diaminopentane by Corynebacterium glutamicum. Appl Environ Microbiol. 2010;76(15):5175-80.

65. Rohles CM, Gießelmann G, Kohlstedt M, Wittmann C, Becker J. Systems metabolic engineering of Corynebacterium glutamicum for the production of the carbon-5 platform chemicals 5-aminovalerate and glutarate. Microb Cell Fact. 2016;15(1)

66. Kallscheuer N, Marienhagen J. Corynebacterium glutamicum as platform for the production of hydroxybenzoic acids. Microb Cell Fact. 2018.

67. Nielsen J, Keasling JD. Engineering cellular metabolism. Cell. 2016

68. Paddon CJ, Keasling JD. Semi-synthetic artemisinin: a model for the use of synthetic biology in pharmaceutical development. Nat Rev Microbiol. 2014.

69. Becker J, Lange A, Fabarius J, Wittmann C. Top value platform chemicals: biobased production of organic acids. Curr Opin Biotechnol. 2015;36:168-75.

70. Karlsson E, Shin JH, Westman G, Eriksson LA, Olsson L, Mapelli V. In silico and in vitro studies of the reduction of unsaturated $a, \beta$ bonds of trans-2-hexenedioic acid and 6-amino-trans-2-hexenoic acid-important steps towards biobased production of adipic acid. PLoS One. 2018;13(2).

71. Paczia N, Nilgen A, Lehmann T, Gätgens J, Wiechert W, Noack S. Extensive exometabolome analysis reveals extended overflow metabolism in various microorganisms. Microb Cell Fact. 2012

72. Hummel J, Strehmel N, Selbig J, Walther D, Kopka J. Decision tree supported substructure prediction of metabolites from GC-MS profiles. Metabolomics. 2010

\section{Publisher's Note}

Springer Nature remains neutral with regard to jurisdictional claims in published maps and institutional affiliations.
Ready to submit your research? Choose BMC and benefit from:

- fast, convenient online submission

- thorough peer review by experienced researchers in your field

- rapid publication on acceptance

- support for research data, including large and complex data types

- gold Open Access which fosters wider collaboration and increased citations

- maximum visibility for your research: over 100M website views per year

At BMC, research is always in progress.

Learn more biomedcentral.com/submissions 